\title{
Generalized Discontinuous Conduction Modes in the Complementarity Formalism
}

\author{
Carles Batlle, Enric Fossas, Iván Merillas, and Alicia Miralles
}

\begin{abstract}
We model dc-dc power converters using the complementarity formalism. For each position of the switches, the dynamics is given by a linear complementarity system which incorporates, in a natural way, the description of generalized discontinuous conduction modes (GDCM), characterized by a reduction of the dimension of the effective dynamics. For systems with a single diode, analytical state-space conditions for the presence of a GDCM can be stated. As an example, this result is used to identify the GDCM for the switch configurations of the Čuk converter. Simulation results, showing a variety of behaviors, such as persistent or re-entering GDCM, are presented.
\end{abstract}

Index Terms-Discontinuous conduction modes (DCMs), linear complementarity systems (LCS), switched power converters.

\section{INTRODUCTION}

$\mathbf{T}$ HIS PAPER studies some characteristics of the dynamical behavior of switched power converters in the framework of linear complementarity systems (LCS). LCS are obtained as follows. Take a standard linear system, select a number of input/output pairs $\left(u_{i}, y_{i}\right)$ and impose for each of these pairs that at each time $t$ both $u_{i}(t)$ and $y_{i}(t)$ must be nonnegative, and at least one of them should be zero (nonnegativity + orthogonality)

$$
u_{i}(t) \geq 0 \quad y_{i}(t) \geq 0 \quad u_{i}(t) y_{i}(t)=0 \quad \forall i \quad \forall t .
$$

These are called the "complementarity conditions" (CC), and are denoted collectively as

$$
0 \leq u \perp y \geq 0 .
$$

The pairs $\left(u_{i}, y_{i}\right)$ are called "complementary variables." CC are well known in mathematical programming [1], although not usually in combination with differential equations. In the context of electrical circuits, imposing complementarity conditions simply means that some ports are terminated by ideal diodes, with the current $i_{D}$ and (minus) the voltage $-v_{D}$ as complementary variables.

Manuscript received July 26, 2004; revised November 18, 2004 and January 24,2005 . This work was supported by the European sponsored Project Siconos IST-2001-37 172. The work of C. Batlle and E. Fossas was supported in part by the MCyT Project DPI2002-03279. The work of A. Miralles was supported by TIC2002-00 155. This paper was recommended by Associate Editor S. Banerjee.

C. Batlle is with the Engineering School of Vilanova i la Geltrú (EPSEVG) and with the Institute of Control and Industrial Engineering (IOC), Technical University of Catalonia (UPC), 08800 Vilanova i la Geltrú, Spain (e-mail: carles.batlle@upc.edu).

E. Fossas is with the Institute of Control and Industrial Engineering (IOC), Technical University of Catalonia (UPC), 08028 Barcelona, Spain.

I. Merillas is with the Department of Applied Mathematics IV, Technical University of Catalonia (UPC), 08028 Barcelona, Spain.

A. Miralles is with the Engineering School of Castelldefels (EPSC), Technical University of Catalonia (UPC), 08800 Vilanova i la Geltrú, Spain.

Digital Object Identifier 10.1109/TCSII.2005.849010
Associated to each complementary pair $\left(u_{i}, y_{i}\right)$ there are two general situations allowed by the CC: either $u_{i}=0$ and $y_{i} \geq 0$ or $y_{i}=0$ and $u_{i} \geq 0$. In electrical engineering terminology, diodes may be blocking or conducting. If there are $p$ diodes, one has $2^{p}$ of these binary choices and the system can be in any of $2^{p}$ so-called "modes". For power converters one has, in addition to (ideal) diodes, some (ideal) switches which are arbitrarily closed or open by a control law. Ideal switches do not dissipate or store power, and hence the product of current and voltage for any of them is zero, $i_{S} v_{S}=0$. This resembles part of a CC; however one does not have, in general, a positiveness condition in this case (although some physical realizations of the switch may impose some kind of partial positiveness; see [2]). The general theory for LCS is covered, for instance in [3]-[7]; the extension of the theory to deal with four-quadrant switches can be found in [8]. In this paper we analyze in detail some aspects of the dynamics of the simplest power converters for a given switch configuration; thus only the changes associated to the diodes are taken into account and the standard LCS framework will be used.

Since some of the complementary variables are linked to state variables by static relations, the evolution of the later can bring some of the former to zero; they cannot decrease any further without violating the $\mathrm{CC}$ and this may force a nonsmooth change in some of the other complementary variables so that the vector field for the state variables takes the correct sign. For an arbitrary number of complementary pairs this process must, in general, be solved numerically, using some well known algorithms, such as Lemke's or Murty's [1], [9], coupled to an implicit integration algorithm, such as the backward Euler scheme [5]. However, for a single pair, i.e., a single diode, analytical results can be obtained and the state space conditions for the above jump to take place can be given in detail. If a complementary variable is nonzero, the complementarity condition forces its conjugate to remain equal to zero for a while, and hence one or more state variables (or combination of them) may be kept to a constant value for a time. This situation, in the case when the variable is a current, is known as a "discontinuous conduction mode" (DCM) in the power converter literature [10]. Since this can happen to a wider class of variables, other than currents, we call this situation "generalized DCM" (GDCM). The GDCM lasts until a switch state change takes place, or until the companion complementary variable returns to zero due to the dynamics of the other state variables. DCMs have been extensively studied in the literature in connection with control algorithms, i.e., switching policies, such as PWM (see [11] and references therein), where averaged methods or small-signal frequency domain descriptions are 


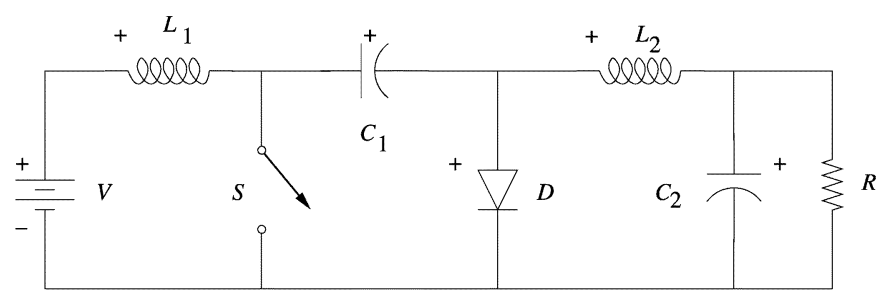

Fig. 1. Čuk converter.

generally used, or in connection to bifurcation theory and chaos [12]. Instead, our aim is to obtain, for a given switch position, an exact state-space condition which indicates the appearance of a GDCM.

The paper is organized as follows. Section II presents the general LCS formulation and then we apply it to the Čuk converter. Section III presents the main results of this paper. In Section IV these results are applied to the Čuk converter for each configuration of the switches. Several simulations, which corroborate the theoretical predictions and display some interesting phenomena, are run. Finally, we summarize our results in Section V and discuss some open questions.

\section{POWER CONVERTERS AS COMPLEMENTARITY Systems}

The general form of an LCS is given by

$$
\begin{aligned}
& \dot{x}=A x+B u+E \\
& y=C x+D u+F
\end{aligned}
$$

with $x \in \mathbb{R}^{n}$. Here $y, u$ are $p$ pairs of complementary variables $0 \leq y \perp u \geq 0$, and $A, B, C, D, E$, and $F$ are (constant) matrices and vector of suitable dimensions. If one allows for the presence of switches, positiveness must be relaxed to various degrees (depending on the detailed implementation of the switches) for the corresponding pairs, and one gets a linear cone complementarity system (LCCS) [8].

To illustrate the LCS description, we apply it to the Čuk converter, but any other power converter can be modeled as well. The basic scheme of the Čuk converter is presented in Fig. 1, and the complementarity description is obtained as follows.

We take as state variables $x_{1}=i_{L 1}, x_{2}=i_{L 2}, x_{3}=v_{C 1}$ and $x_{4}=v_{C 2}$, and $u_{1}=-v_{D}, u_{2}=i_{S}, y_{1}=i_{D}=x_{1}-x_{2}-u_{2}$ and $y_{2}=v_{S}=x_{3}-u_{1}$ as complementary variables. Then, in matrix notation

$$
\left\{\begin{array}{l}
\dot{x}=A x+B u+E V \\
y=C x+D u+F V \\
0 \leq y_{1} \perp u_{1} \geq 0 \\
y_{2} \perp u_{2}
\end{array}\right.
$$

with

$$
\begin{aligned}
& A=\left(\begin{array}{cccc}
0 & 0 & -\frac{1}{L_{1}} & 0 \\
0 & 0 & 0 & -\frac{1}{L_{2}} \\
\frac{1}{C_{1}} & 0 & 0 & 0 \\
0 & \frac{1}{C_{2}} & 0 & -\frac{1}{R C_{2}}
\end{array}\right) \\
& B=\left(\begin{array}{cc}
\frac{1}{L_{1}} & 0 \\
-\frac{1}{L_{2}} & 0 \\
0 & -\frac{1}{C_{1}} \\
0 & 0
\end{array}\right)
\end{aligned}
$$

$$
\begin{aligned}
C & =\left(\begin{array}{cccc}
1 & -1 & 0 & 0 \\
0 & 0 & 1 & 0
\end{array}\right) \\
D & =\left(\begin{array}{cc}
0 & -1 \\
-1 & 0
\end{array}\right) \\
E & =\left(\begin{array}{l}
\frac{1}{L_{1}} \\
0 \\
0 \\
0
\end{array}\right) \\
F & =\left(\begin{array}{l}
0 \\
0
\end{array}\right) .
\end{aligned}
$$

\section{Generalized Discontinuous-Conduction Modes FOR SYSTEMS With SINGLE DiOdE}

Our approach to computing conditions for the presence of a GDCM is based on ideas presented in [3]. It is based on assuming right-analyticity of solutions in state-space. This means that any quantity can be computed on $\left(t_{0}, t_{0}+\epsilon\right)$, for some $\epsilon>0$, if the quantity and its derivatives are known in $t_{0}$. To be more precise, let us consider a couple of complementary variables, $u$ and $y$, and let $\mathcal{U} \equiv\left(u^{(0)}, u^{(1)}, u^{(2)}, \ldots\right), \mathcal{Y}=$ $\left(y^{(0)}, y^{(1)}, y^{(2)}, \ldots\right)$ denote the values of $u$ and $y$ and their successive right-time derivatives at $t=t_{0}$. To ensure $0 \leq u \perp y \geq$ 0 on $\left[t_{0}, t_{0}+\epsilon\right)$, one of the following must be true:

$$
\mathcal{U} \succeq 0 \text { and } \mathcal{Y}=0
$$

or

$$
\mathcal{Y} \succeq 0 \text { and } \mathcal{U}=0
$$

where $\succeq$ means lexicographic nonnegativity [9], i.e., all the terms are zero or the first nonzero term is positive. If the terms of the sequence are the Taylor coefficients of an analytic function, lexicographic nonnegativity ensures nonnegativity of the function in an open interval. We will also consider finite sequences $\mathcal{U}_{k} \equiv\left(u^{(0)}, u^{(1)}, \ldots, u^{(k)}\right), \mathcal{Y}_{k}=\left(y^{(0)}, y^{(1)}, \ldots, y^{(k)}\right)$. A pair of sequences, finite or not, satisfying either (4) or (5), will be called valid.

Consider a dynamical system of the form

$$
\begin{aligned}
\dot{x} & =f(x, z)+a u \\
\dot{z} & =g(x, z, u) \\
y & =\beta x+\alpha,
\end{aligned}
$$

with $x \in \mathbb{R}, z \in \mathbb{R}^{n-1}, a \neq 0, \beta \neq 0$, and where $u \in \mathbb{R}$ and $y \in \mathbb{R}$ are complementary variables, $0 \leq u \perp y \geq 0$. This is a relative degree $\rho=1$ system, and from (6), (7), and (8) one can compute the equations linking the values of $u, y$ and their successive time derivatives at $t=t_{0}$. Using the notation explained above, the first of these are

$$
\begin{aligned}
y^{(0)}= & \beta x_{0}+\alpha \equiv \beta \gamma_{0} \\
y^{(1)}= & \beta f\left(x_{0}, z_{0}\right)+a \beta u^{(0)} \equiv \beta \gamma_{1}+a \beta u^{(0)} \\
y^{(2)}= & \beta \partial_{x} f\left(x_{0}, z_{0}\right)\left(f\left(x_{0}, z_{0}\right)+a u^{(0)}\right) \\
& +\beta \partial_{z} f\left(x_{0}, z_{0}\right) g\left(x, z, u^{(0)}\right)+a \beta u^{(1)} \\
\equiv & \beta \gamma_{2}+a \beta u^{(1)}
\end{aligned}
$$

and, in general

$$
y^{(k)}=\beta \gamma_{k}+a \beta u^{(k-1)}
$$


where $\gamma_{k}$ depends on $x_{0}, z_{0}$ and the time derivatives of $u$ at $t=t_{0}$ up to order $k-2$.

As explained in [3], a method for constructing smooth solutions starting from $x_{0}, z_{0}$ can be obtained by solving a series of what are called there dynamical complementarity problems (DCP). A DCP $(k)$ consists in finding valid sequences $\mathcal{U}_{k}$ and $\mathcal{Y}_{k}$ satisfying relations (9), (10), . . up to (12). In general, DCP $(k)$ may have many solutions (for instance, $u^{(0)}$ does not appear in (9), and hence it is free, apart from being nonnegative, for DCP (0)); however, since the conditions of DCP $(k)$ are a subset of those of DCP $(k+1)$, the solutions of $\mathrm{DCP}(k+1)$ must be chosen among those of DCP $(k)$. This is called the nesting property of the DCP.

We say that the system is in mode $J_{k}$ if all the solutions of $\operatorname{DCP}(k)$ satisfy $\mathcal{Y}_{k} \succ 0$, and that the system is in mode $I_{k}$ if all the solutions of $\operatorname{DCP}(k)$ verify $\mathcal{U}_{k} \succ 0$; otherwise, the system is said to be in mode $K_{k}{ }^{1}$. Due to the nesting property, if the system is in mode $J_{k}\left(I_{k}\right)$ it will be in mode $J_{l}$ (resp. $I_{l}$ ) for any $l>k$. We will assume that:

1) $y^{(0)} \geq 0$

2) $a \beta>0$.

These conditions ensure in our case existence and uniqueness of smooth solutions starting from $x_{0}, z_{0}$ [3,Th. 3.1, 3.2 ]. Then, the DCPs are as follows.

$\operatorname{DCP}(\mathbf{0})$ : Find a valid pair $\left(u^{(0)}\right),\left(y^{(0)}\right)$ such that (9) holds. Two situations are possible.

Case 1) $y^{(0)}>0$. This forces $u^{(0)}=0$. The system is in mode $J_{0}$.

Case 2) $y^{(0)}=0$. In this case $u^{(0)} \geq 0$ is still free. The system is in mode $K_{0}$.

$\operatorname{DCP}(1)$ : Find a valid pair $\left(u^{(0)}, u^{(1)}\right),\left(y^{(0)}, y^{(1)}\right)$ such that (9) and (10) hold. Since the conditions of $\operatorname{DCP}(0)$ are a subset of these, we start with the solutions obtained there.

Case 1) Since $y^{(0)}>0$ we must have $u^{(1)}=0$ for the pair to be valid. One also gets $y^{(1)}=\beta \gamma_{1}$. Notice that the sign of $y^{(1)}$ does not matter since already $y^{(0)}>0$. The system is in mode $J_{1}$.

Case 2) In this case (10) becomes a 1-dimensional LCP for $y^{(1)}, u^{(0)}$, which always has solution due to $a \beta>0$. Three subcases are possible.

Case 2.1) $\quad \beta \gamma_{1}>0$. The only solution to the LCP is $u^{(0)}=$ $0, y^{(1)}=\beta \gamma_{1}>0$; one must choose $u^{(1)}=0$ and the system is in mode $J_{1}$.

Case 2.2) $\quad \gamma_{1}=0$. Now we have $u^{(0)}=0, y^{(1)}=0$; any $u^{(1)} \geq 0$ is valid and the system is in mode $K_{1}$.

Case 2.3) $\beta \gamma_{1}<0$. The solution is $u^{(0)}=-\left(\gamma_{1} / a\right)=$ $-\left(\beta \gamma_{1}\right) /(a \beta)>0, y^{(1)}=0$; any $u^{(1)} \in \mathbb{R}$ is valid and the system is in mode $I_{1}$.

$\mathbf{D C P}(2)$ : Find a valid pair $\left(u^{(0)}, u^{(1)}, u^{(2)}\right),\left(y^{(0)}, y^{(1)}, y^{(2)}\right)$ such that (9), (10), and (11) hold. As is the transition to $\operatorname{DCP}(0)$ to $\mathrm{DCP}(1)$, solutions coming from modes $I_{1}$ or $J_{1}$ will yield solutions in $I_{2}$ and $J_{2}$, respectively, so the only case worth studying is 2.2, for which now (11) is an LCP for $y^{(2)}, u^{(1)}$. Again this always has solution due to $a \beta>0$, and three situations can be encountered:

\footnotetext{
${ }^{1}$ We use the term mode in a sense different from that of [3]; ours is adapted to the fact that only a pair of complementary variables are present.
}

Case 2.2.1) $\beta \gamma_{2}>0$. The solution is $u^{(1)}=0, y^{(2)}=$ $\beta \gamma_{2}>0$; one must choose $u^{(2)}=0$ and the system is in mode $J_{2}$.

Case 2.2.2) $\gamma_{2}=0$. Now $u^{(1)}=0, y^{(2)}=0$; any $u^{(2)} \geq 0$ is valid and the system is in mode $K_{2}$.

Case 2.2.3) $\beta \gamma_{2}<0$. The solution is $u^{(1)}=-\left(\gamma_{2}\right) /(a)=$ $-\left(\beta \gamma_{2}\right) /(a \beta)>0, y^{(2)}=0$; any $u^{(2)} \in \mathbb{R}$ is valid and the system is in mode $I_{2}$.

Successive DCPs can be solved, and we assume that after a finite number of steps the system ends up in a $J$ or $I$ mode. The general case can be summarized as follows:

Proposition 1: Under the conditions of the preceding discussion the following are true.

- If the above procedure enters a $J$ mode for the first time when solving $\operatorname{DCP}(k)$, then $u^{(l)}=0$ for all $l \in \mathbb{N}, y^{(l)}=$ 0 for $l=0, \ldots, k-1$, and $y^{(k)}=\beta \gamma_{k}>0$.

- If the above procedure enters a $I$ mode for the first time when solving $\operatorname{DCP}(k)$, then $y^{(l)}=0$ for all $l \in \mathbb{N}, u^{(l)}=$ 0 for $l=0, \ldots, k-2$, and $u^{(k-1)}=-\left(\gamma_{k}\right) /(a)>0$.

Proposition 2: Assume that $y(t)>0$, and hence $u(t)=0$, for $t \in\left(t_{0}-\tilde{\epsilon}, t_{0}\right)$, for some $\tilde{\epsilon}>0$. Then, if for some $k \geq 1$

$\gamma_{0}=0, \quad$ and $\quad \gamma_{l}=0, \forall l=1, \ldots, k-1, \quad$ but $\quad \beta \gamma_{k}<0$

one has the following.

- The $(k-1)$ th time derivative of $u$ has a jump at $t_{0}$, going from 0 to $-\gamma_{k} / a>0$.

- There exists $\epsilon>0$ such that $y(t)=0, u(t)>0$ for $t \in\left(t_{0}, t_{0}+\epsilon\right)$, and hence we have a GDCM.

We call (13) the $k$ th-order GDCM condition (GDCMC).

Proof: Obvious from Proposition 1 and the discussion leading to it, and the fact that all the derivatives of $u$ are zero for $t \in\left(t_{0}-\tilde{\epsilon}, t_{0}\right)$.

As a corollary, we have the following.

Proposition 3: Assume $y(t)>0$ for $t \in\left(t_{0}-\tilde{\epsilon}, t_{0}\right)$, for some $\tilde{\epsilon}>0$, and that $y\left(t_{0}\right)=0$ and $\beta \gamma_{1}<0$. Then $u$ has a discontinuity at $t=t_{0}$, from 0 to $-\gamma_{1} / a>0$, and there exists $\epsilon>0$ such that $y(t)=0$ and $u(t)>0$ for $t \in\left(t_{0}, t_{0}+\epsilon\right)$.

The situation presented in Proposition 3 is the one normally encountered both in simulation and in experiment; higher order GDCM conditions are difficult to meet, since they require several state space quantities to be zero simultaneously.

\section{APPliCATION TO THE ČUK CONVERTER}

In this section, we will obtain the first-order GDCMC for the two positions of the switch of the Čuk converter.

Setting $v_{S}=0$ or $i_{S}=0$ in (3) one gets the following two LCS.

1) $S$ Closed $\left(v_{S}=0\right)$ :

$$
\begin{aligned}
L_{1} \dot{x}_{1} & =V \\
L_{2} \dot{x}_{2} & =-x_{3}-x_{4} \\
C_{1} \dot{x}_{3} & =x_{2}+i_{D} \\
C_{2} \dot{x}_{4} & =x_{2}-\frac{1}{R} x_{4} \\
x_{3} & =-v_{D} .
\end{aligned}
$$


We apply Proposition 3 with $x=x_{3}, z=\left(x_{1}, x_{2}, x_{4}\right), u=$ $i_{D}, y=-v_{D}, f(x, z)=x_{2} / C_{1}, \alpha=0, \beta=1, a=1 / C_{1}$. The first-order GDCMC is given by

$$
x_{3}\left(t_{0}\right)=0, \quad \gamma_{1}=\frac{1}{C_{1}} x_{2}\left(t_{0}\right)<0 .
$$

This implies $x_{2}\left(t_{0}\right)<0$. On $x_{3}(t)=0$ one can prove that $u$ obeys

$$
\ddot{u}+\frac{1}{R C_{2}} \dot{u}+\frac{1}{L_{2} C_{2}} u=0
$$

which has oscillating solutions for usual values of the parameters. Hence, $u$ reaches zero in finite time and the GDCM disappears.

2) $S$ Open $\left(i_{S}=0\right)$ :

$$
\begin{aligned}
L_{1} \dot{x}_{1} & =-x_{3}-v_{D}+V \\
L_{2} \dot{x}_{2} & =-x_{4}+v_{D} \\
C_{1} \dot{x}_{3} & =x_{1} \\
C_{2} \dot{x}_{4} & =x_{2}-\frac{1}{R} x_{4} \\
i_{D} & =x_{1}-x_{2} .
\end{aligned}
$$

Notice that, if $x=x_{1}-x_{2}$, then

$$
\dot{x}=\frac{V}{L_{1}}-\frac{1}{L_{1}} x_{3}+\frac{1}{L_{2}} x_{4}-\left(\frac{1}{L_{1}}+\frac{1}{L_{2}}\right) v_{D} .
$$

Thus, we can apply Proposition 3 with $x=x_{1}-x_{2}, z=\left(x_{1}+\right.$ $\left.x_{2}, x_{3}, x_{4}\right), u=-v_{D}, y=i_{D}, f(x, z)=V / L_{1}-x_{3} / L_{1}+$ $x_{4} / L_{2}, \alpha=0, \beta=1, a=1 / L_{1}+1 / L_{2}$.

The first-order GDCMC is given by

$$
x_{1}\left(t_{0}\right)=x_{2}\left(t_{0}\right), \quad \gamma_{1}=\frac{V}{L_{1}}-\frac{x_{3}\left(t_{0}\right)}{L_{1}}+\frac{x_{4}\left(t_{0}\right)}{L_{2}}<0 .
$$

A detailed study shows that the GDCM can have in this case either a finite or unbounded duration, depending on the initial conditions.

As shown in [5], a backward Euler integration method yields a stable algorithm when complementarity conditions are present. For a general LCS (1), (2), the backward Euler scheme is

$$
\begin{aligned}
& x_{k+1}=x_{k}+h\left(A x_{k+1}+B u_{k+1}+E\right) \\
& y_{k+1}=C x_{k+1}+D u_{k+1}+F
\end{aligned}
$$

where $h$ is the time step size. At each step, one must solve the LCP

$$
\begin{aligned}
& y_{k+1}=\left(h C(\mathbb{\square}-h A)^{-1} B+D\right) u_{k+1} \\
& +C(\mathbb{\square}-h A)^{-1} x_{k} \\
& +h C(\mathbb{\square}-h A)^{-1} E+F \\
& 0 \leq y_{k+1} \perp u_{k+1} \geq 0
\end{aligned}
$$

for given $x_{k}$, and then compute

$$
x_{k+1}=(\mathbb{\square}-h A)^{-1}\left(x_{k}+h\left(B u_{k+1}+E\right)\right)
$$

from the obtained value of $u_{k+1}$. In general, an LCP must be solved using specialized algorithms (such as Lemke's [1]). However, for a single pair of complementary variables, an explicit computation can be done. First of all, (14) can be
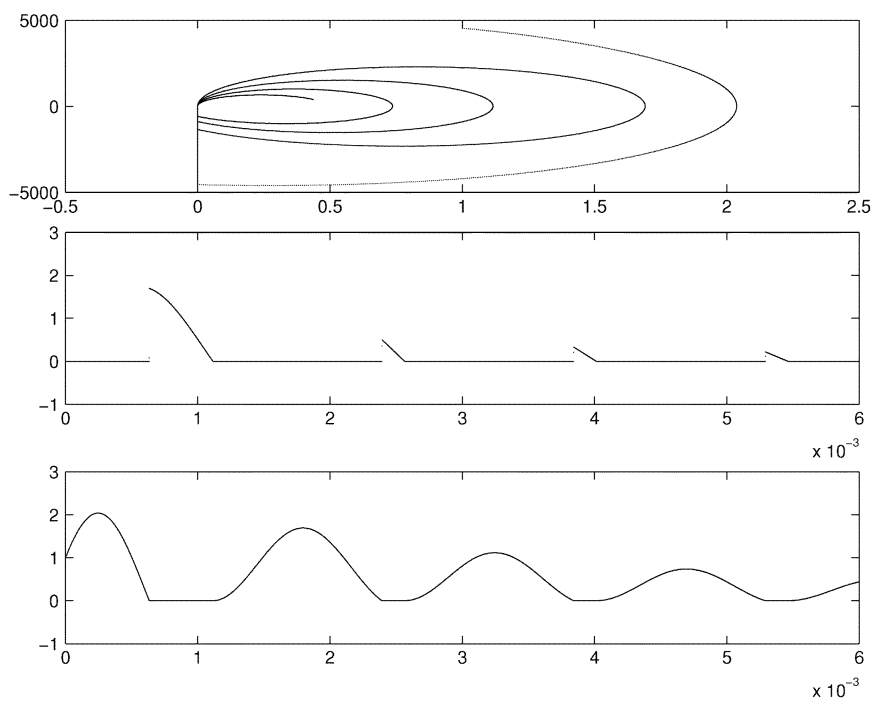

Fig. 2. GDCM for Čuk converter with switch closed. Upper: $x_{3}$ on the horizontal axis and $\Gamma$ on the vertical one. Middle: $u$ as a function of time. Lower: $y$ as a function of time. The GDCM has a finite duration, but it is re-entrant for the parameters and initial conditions chosen.

written as $y_{k+1}=M u_{k+1}+q_{k}$. Assume $M>0$ (which is the case for any of the switch configurations of our converters). Then the LCP is solved as follows.

- $\quad$ if $q_{k}>0$, then $u_{k+1}=0$ and $y_{k+1}=q_{k}$,

- $\quad$ if $q_{k}<0$, then $u_{k+1}=-q_{k} / M$ and $y_{k+1}=0$,

- $\quad$ if $q_{k}=0$, then $u_{k+1}=0$ and $y_{k+1}=0$.

We have implemented this algorithm in Mat lab to check the GDCMC for the Čuk converter. We have used the system parameter values (in SI units) $L_{1}=750 \cdot 10^{-6}, L_{2}=800 \cdot 10^{-6}, C_{1}=$ $220 \cdot 10^{-6}, C_{2}=130 \cdot 10^{-6}, R=10$ and $V=24$. The initial conditions are $x(0)=(2,1,1,1)$ for both simulations of the Čuk converter, while the fixed integration step has been chosen as $h=10^{-6}$.

Simulation results are displayed in Figs. 2 and 3. $\Gamma$ is $\gamma_{1}$ computed on an arbitrary state-space point, not just at the one corresponding to $t_{0}$. Notice that, as predicted, $\Gamma<0$ when entering the GDCM. For the Čuk converter with switch closed, the GDCM is entered multiple times, while for open switch the system does not leave the GDCM, although a re-entrant behavior can be obtained if other initial conditions are used.

\section{CONCLUSION}

The basic power converters can be formulated as LCS, with the positivity condition relaxed. For a given switch configuration, the resulting system can be cast strictly in the LCS formalism.

We have presented a simple analytical test to look for GDCMs in power converters with a single diode. This test can be verified at several orders; the higher the order, the smoother the change in $u$. We have applied the test to the Čuk converter, and found the conditions under which GDCMs can appear. We have performed simulations and checked the theoretical prediction. In particular, we have found a variety of behaviors, such as a 

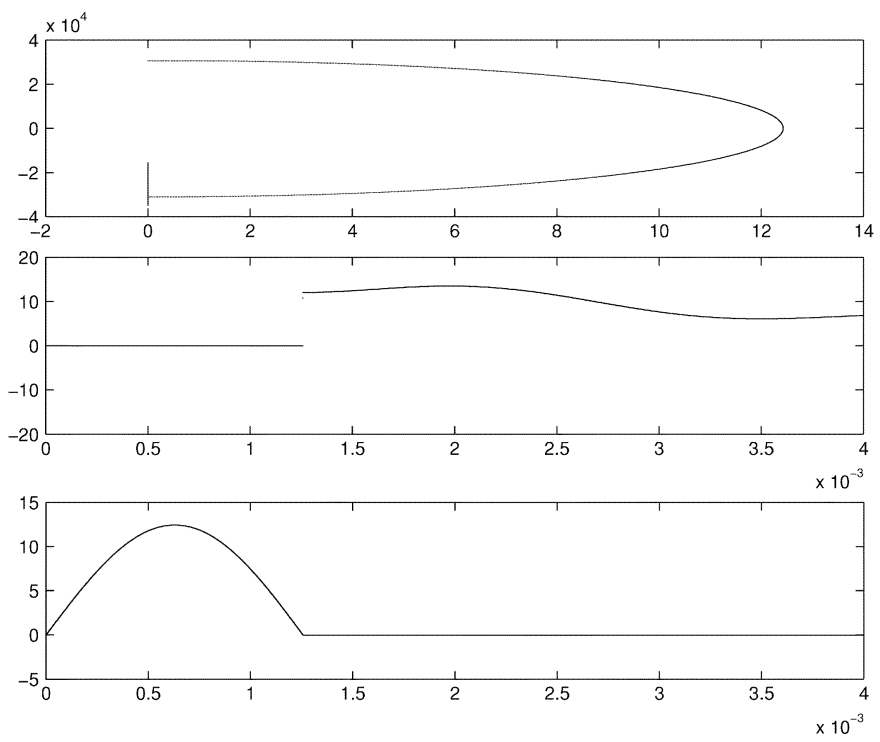

Fig. 3. GDCM for Čuk converter with switch open. Upper: $x_{1}-x_{2}$ on the horizontal axis and $\Gamma$ on the vertical one. Middle: $u$ as a function of time. Lower: $y$ as a function of time. For the parameters and initial conditions used, the GDCM lasts indefinitely.

re-entrant GDCM. Although we have centered our exposition on switched power converters, the results apply as well to any other LCS with a single complementary pair.

Using the formalism of [3] as has been applied here, systems with several diodes can be treated. It is straightforward to extend the study to the case of decoupled diodes, i.e., to the case when $\beta a$, now a matrix, is diagonal; for the nondiagonal case, a case-by-case study of the LCP problems appearing in the successive DCPs will be needed to obtain analytical results, although numerical algorithms can always be used.

\section{ACKNOWLEDGMENT}

The authors would like to thank Prof. J. L. Rodríguez Marrero for his comments on a preliminary version of this work.

\section{REFERENCES}

[1] S. Billups and K. Murty, "Complementarity problems," J. Comput. Appl. Math., vol. 124, pp. 303-318, 2000.

[2] M. Çamlibel, L. Iannelli, and F. Vasca, "Modeling switching power converters as complementarity systems," in Proc. 43rd IEEE Conf. Decision and Control, 2004, pp. 2328-2333.

[3] A. van der Schaft and J. Schumacher, "Complementarity modeling of hybrid systems," IEEE Trans. Autom. Contr., vol. 43, no. 4, pp. 483-490, Apr. 1998.

[4] D. Leenaerts and W. van Bokhoven, Piecewise Linear Modeling and Analysis. Norwell, MA: Kluwer, 1998.

[5] M. Çamlibel, "Complementarity methods in the analysis of piecewise linear dynamical systems," Ph.D. dissertation, Tilburg Univ., Tilburg, The Netherlands, 2001.

[6] W. Heemels, M. Çamlibel, and J. Schumacher, "On the dynamic analysis of piecewise-linear networks," IEEE Trans. Circuits Syst. I, Fundam. Theory Appl., vol. 49, no. 3, pp. 315-327, Mar. 2002.

[7] M. Çamlibel, W. Heemels, and J. Schumacher, "On linear passive complementarity systems," Eur. J. Contr., vol. 8, pp. 220-237, 2002.

[8] M. Çamlibel, W. Heemels, A. van der Schaft, and J. Schumacher, "Switched networks and complementarity," IEEE Trans. Circuits Syst. I, Fundam. Theory Appl., vol. 50, no. 8, pp. 1036-1046, Aug. 2003.

[9] R. Cottle, J.-S. Pang, and R. Stone, The Linear Complementarity Problem. New York: Academic, 1992.

[10] R. Erickson, Fundamentals of Power Electronics. Norwell, MA: Kluwer, 1997.

[11] A. Reatti and M. Kazimierczuk, "Small-signal model of PWM converters for discontinuous conduction mode and its application for boost converter," IEEE Trans. Circuits Syst. I, Fundam. Theory Appl., vol. 50, no. 1, pp. 65-73, Jan. 2003.

[12] S. Parui and S. Banerjee, "Bifurcations due to transition from continuous conduction mode to discontinuous conduction mode in the boost converter," IEEE Trans. Circuits Syst. I, Fundam. Theory Appl., vol. 50, no. 11, pp. 1464-1469, Nov. 2003. 